\title{
Staged implant placement after defect regeneration using biphasic calcium phosphate materials with different surface topographies in a minipig model
}

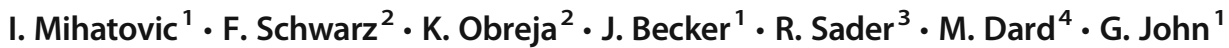

Received: 26 August 2019 / Accepted: 7 January 2020 / Published online: 24 January 2020

(C) The Author(s) 2020

\begin{abstract}
Objective To assess the influence of biphasic calcium phosphate materials with different surface topographies on bone formation and osseointegration of titanium implants in standardized alveolar ridge defects.

Materials and methods Standardized alveolar ridge defects $(6 \times 6 \mathrm{~mm})$ were created in the mandible of 8 minipigs and filled with three biphasic calcium phosphate materials (BCP1-3, 90\% tricalcium phosphate/10\% hydroxyapatite) with different surface properties (micro- and macroporosities) as well as a bovine-derived natural bone mineral (NBM) as a control. At 12 weeks, implants were placed into the augmented defects. After further 8 weeks of healing, dissected blocks were processed for histological analysis (e.g., mineralized (MT), residual bone graft material (BS), bone-to-implant contact (BIC)).

Results All four biomaterials showed well-integrated graft particles and new bone formation within the defect area. MT values were comparable in all groups. BS values were highest in the NBM group $(21.25 \pm 13.52 \%)$ and markedly reduced in the different BCP groups, reaching statistical significance at BCP1-treated sites $(9.2 \pm 3.28 \%)$. All test and control groups investigated revealed comparable and statistically not significant different BIC values, ranging from $73.38 \pm 20.5 \%$ (BCP2) to $84.11 \pm$ $7.84 \%$ (BCP1), respectively.
\end{abstract}

Conclusion All bone graft materials facilitated new bone formation and osseointegration after $12+8$ weeks of healing.

Keywords Bone remodelling $\cdot$ Bone substitute $\cdot$ Histological analysis $\cdot$ Implant osseointegration $\cdot$ Animal model

\section{Introduction}

Bone grafting has become an important field in implant dentistry for the treatment of alveolar ridge defects due to trauma,

Ilja Mihatovic and Karina Obreja equally contributed to the present study and are considered joint first-authors.

F. Schwarz

f.schwarz@med.uni-frankfurt.de

1 Department of Oral Surgery and Central Admittance, University Hospital, Heichrich-Heine-University, Düsseldorf, Germany

2 Department of Oral Surgery and Implantology, Carolinum, Goethe University, Frankfurt, Germany

3 Department for Oral, Cranio-Maxillofacial and Facial Plastic Surgery, Medical Center of the Goethe University Frankfurt, Frankfurt am Main, Germany

4 Section of Oral, Diagnostic and Rehabilitation Sciences, Columbia University, College of Dental Medicine, New York, USA periodontal disease, or infection. Over the years, different biomaterials were used to fill and regenerate bone defects, subsequently allowing an implant-based prosthetic reconstruction. The ideal grafting material should (1) be osteoconductive to serve as a matrix for vascular and cellular migration, (2) feature an osteoinductive potential by stimulating mesenchymal cells to differentiate into bone-forming osteoblasts, and (3) contain osteoprogenitor cells being able to produce new bone matrix. Autogenous bone $(\mathrm{AB})$ comprises all desired properties and represents the gold standard of bone grafts. However, disadvantages of AB like donor site morbidity, limited availability, and graft resorption led to the introduction of alternatives such as allografts, xenografts, and synthetic alloplasts, which include hydroxyapatite, $\beta$-tricalcium phosphates, and biphasic calcium phosphates (BCP) [1-4].

Experimental data indicated that the application of either a particulate xenograft or BCP showed predictable bone formation at chronic alveolar ridge defects in the posterior mandible revealing a vertical and horizontal bone deficiency $[5,6]$. In particular, these experimental data demonstrated that a 
particulate xenograft or BCP with and without the addition of $\mathrm{AB}$ at saddle-type defects were associated with adequate bone formation and osseointegration of titanium implants $[5,6]$. Clinical findings could also support the experimental data reporting a clinically important bone gain $[1,3,7]$. While $\beta$ tricalcium phosphate is fast resorbing and lacks volume stability over time, hydroxyapatite (HA) is very slowly resorbing and might lead to higher amounts of residual graft material to the disfavor of new bone formation [8]. The ideal bone substitute material should be completely resorbable while having a timeframe of resorption stability to maintain space within the defect [9]. The use of biphasic combinations of HA and $\beta$ TCP showed a balance between long-term stability and new bone formation combining the positive features of both materials [10]. Apart from resorption behavior, surface topography of biomaterials seems to play an important role for new bone formation. While macroscale features such as interconnected macropores, particle size and surface concavities have been speculated to be a prerequisite for bone formation, surface microtopography might even influence the osteoinductive capability of certain biomaterials [11-15]. Various experimental models demonstrated that microstructured HA and BCP induced ectopic bone formation [12, 13, 16, 17]. Previously, the dimension of the surface microstructure has also been described to play an important role for osteoinduction. In particular, experimental studies revealed that TCP with a submicron-scale surface structure stimulated de novo bone formation, while TCP with a micron-scale surface did not show any osteoinductive potential $[18,19]$. Based on this data, it might be hypothesized that the surface topography of graft materials, especially microporosity, could have a potential to support new bone formation. Therefore, the aim of the present study was to evaluate the influence of three BCP graft materials with different surface topographies on new bone formation and osseointegration of titanium implants in an experimental defect model.

\section{Material and methods}

\section{Animals}

A total of 8 female Gottingen minipigs (Ellegaard, Dalmose, Denmark) with an age of 20-24 months and a weight of 36$42 \mathrm{~kg}$ were recruited for the present study. During the experiment, minipigs were fed using a standard diet (soft food) expanded for minipigs (SDS Standard Service, UK) and water ad libitum. The animals were kept in specially designed areas under supervision of veterinarian staff during the entire study period. Animal selection, management, and surgery protocol were approved by the Malmö-Lund University Animal Experiment Ethics Committee (number M-204-11.7; Malmö-Lund University, Lund, Sweden). The experimental phase of the study started after an adaption period of 1 week. On the day of surgery, animals were fasted and weighed. Premedication was performed by means of an intramuscular injection atropine $(0.05 \mathrm{mg} / \mathrm{kg})$ of $10 \mathrm{ml}$ ketamine (Ketalar Vet, Pfizer AB, Sollentuna, Sweden; $50 \mathrm{mg} / \mathrm{ml}$ ) mixed with $3 \mathrm{ml}$ midazolam (Dormicum $5 \mathrm{mg} / \mathrm{ml}$ : Roche, Basel, Switzerland). During surgery, $10 \mathrm{ml}$ of ketamine was additionally injected every 30 to $40 \mathrm{~min}$ to maintain sufficient anaesthesia.

\section{Study design and randomization}

The study was performed in three surgical phases (Fig. 1). In the first phase, the lower premolars PM2 -4 as well as the first molars were extracted and the sites were left to heal for a period of 3 months. In the second phase, a total of three standardized cylindrical-type defects (mesio-distal width, $6 \mathrm{~mm}$; height, $6 \mathrm{~mm}$ ) were bilaterally prepared in the lower jaws (i.e., $n=6$ defects per animal).

The following groups were investigated:

- $\quad$ Test 1: BCP1—porous biphasic calcium phosphate (HA 10\%, $\beta$-TCP 90\%/particle size 250-1000 $\mu \mathrm{m}$ (Institut Straumann AG, Basel, Switzerland)

- Test 2: BCP2 - porous biphasic calcium phosphate (HA 10\%, $\beta$-TCP 90\%/particle size 250-1000 $\mu \mathrm{m}$, less macro-/less microporosity (Institut Straumann AG)

- Test 3: BCP3 - porous biphasic calcium phosphate (HA $10 \%$, $\beta$-TCP 90\%), particle size 250-1000 $\mu \mathrm{m}$, more macro-/less microporosity (Institut Straumann AG)

- Control 1: NBM-natural bovine bone material (HA) (BioOss, Geistlich, Wolhusen, Switzerland)

- Control 2: no defect fill

Macroporosity was defined as pores $>75 \mu \mathrm{m}$, and microporosity as pores $>2 \mathrm{~nm}$.

The experimental sites $(n=48)$ were randomized according to a computer-generated list: $\mathrm{BCP} 1(n=10)$; $\mathrm{BCP} 2(n=10)$; BCP3 $(n=10)$; control $1(n=10)$; control $2(n=8)$.

The second healing period was 12 weeks. In the third phase, titanium implants were placed at the respective test and control sites. Following another healing period of 8 weeks, animals were terminated, and separated block biopsies prepared for histological processing (Fig. 1).

\section{Surgical phases}

All surgeries were performed in an operation room ensuring aseptic conditions. Surgeries were conducted under general anaesthesia utilizing an intramuscular injection of $10 \mathrm{ml}$ ketamine (Ketalar Vet, Pfizer AB, Sollentuna, Sweden, $50 \mathrm{mg} / \mathrm{ml}$ ) mixed with $3 \mathrm{ml}$ midazolam (Dormicum® $5 \mathrm{mg} / \mathrm{ml}$; Roche, Basel, Switzerland). During surgery, $10 \mathrm{ml}$ of ketamine was 
Fig. 1 Study outline including tooth extraction, defect augmentation, and implant placement (P, posterior; $\mathrm{M}$, middle; $\mathrm{A}$, anterior defects)

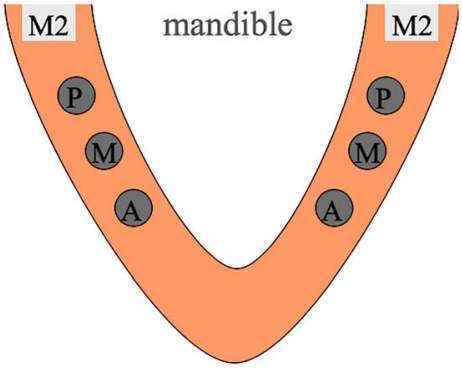

additionally injected every 30 to 40 min to maintain sufficient anaesthesia. While anaesthetized, all animals received a constant rate infusion of lactated Ringer's solution to maintain hydration. The tooth extraction surgeries were performed by three surgeons, whereas defect creation, augmentation, and implant placement were performed by one blinded surgeon.

\section{Tooth extraction}

Following local anaesthesia using $2 \%$ xylocain-adrenaline (ASTRA, Södertälje, Sweden) and midcrestal gingival incision, full-thickness mucoperiostal flaps were raised bilaterally in the mandible. Lower PM2-4 and the first molar M1 were carefully extracted (Fig. 2a). After accurate inspection of the sockets, wound closure was achieved using multiple single sutures (Vicryl 4-0, FS2, Ethicon, Somerville, NJ, USA). Postoperatively, radiographs were taken to control the extraction sites.

\section{Defect creation and augmentation}

After a healing period of 3 months, edentulous mandibular alveolar ridges were exposed bilaterally using midcrestal incisions and reflection of full-thickness flaps. Subsequently, the crestal area was evened out by means of a carbide bur under continuous cooling with saline to achieve a crestal ridge width of at least $10 \mathrm{~mm}$. Three cylindrical-type defects measuring $6 \mathrm{~mm}$ in width and depth were created using spiral burs with ascending diameters. Between each defect and the second molar, a distance of $5 \mathrm{~mm}$ was kept. Additionally, titanium miniscrews $(1.5 \times 6 \mathrm{~mm}$; Medartis AG, Basel, Switzerland) were inserted at the alveolar crest $5 \mathrm{~mm}$ mesial of the most anterior defect to ensure orientation and precise implant placement into the defects at re-entry (Fig. 2b). Following adequate rinsing of the drill holes to remove bone debris, respective defects were homogeneously filled with BCP1, BCP2, BCP3, or NBM (Fig.
Fig. 2 Surgical procedures. a Occlusal view of the alveolar ridge after tooth extraction. $\mathbf{b}$ Occlusal view of the alveolar ridge after creation of cylindricaltype defects. Mesial of the most anterior defect, a miniscrew is visible. c Occlusal view of the alveolar ridge after application of the bone graft material. d Radiographic image after implant placement into the previously augmented defects

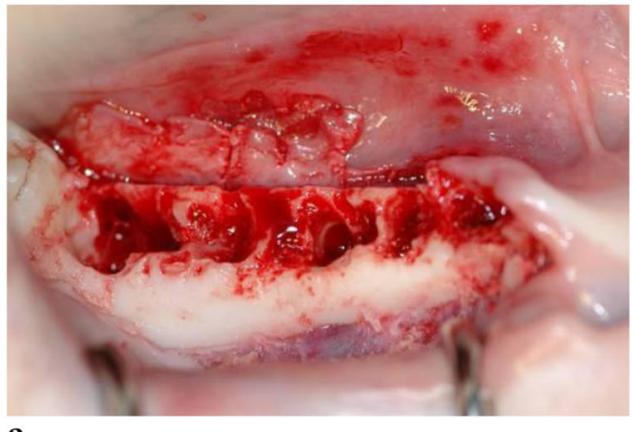

$\mathbf{a}$
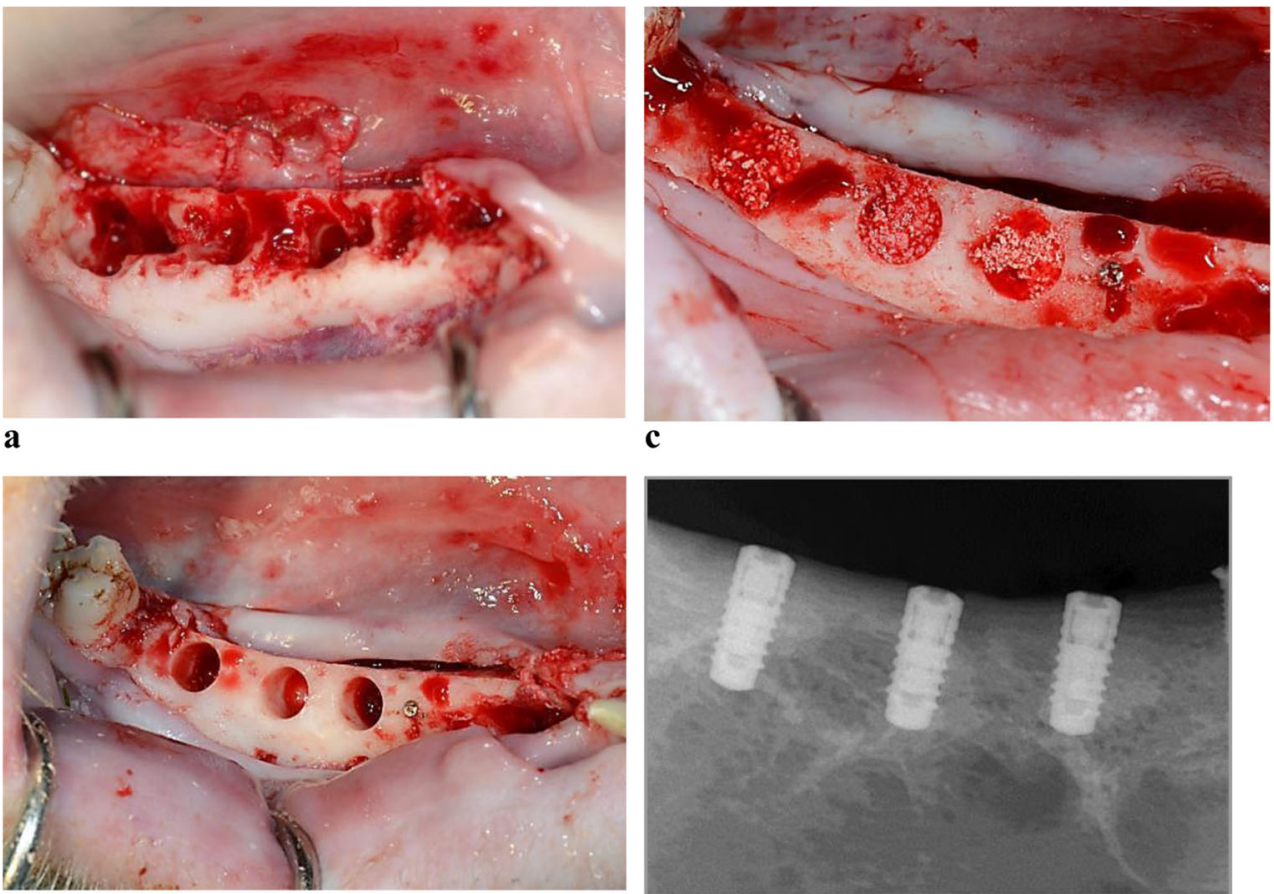

b c

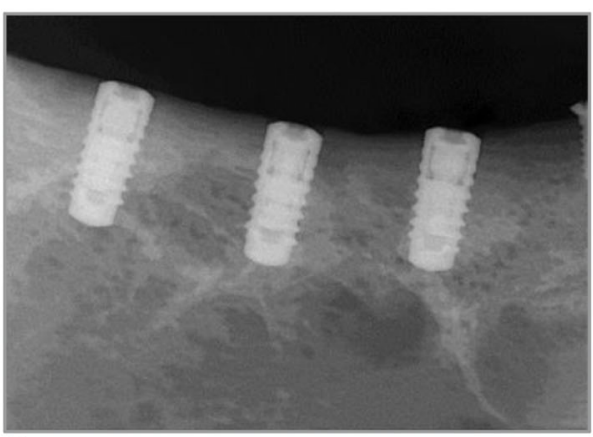

d 
2c). Particular care was taken that the graft particles did not exceed the contour of adjacent bone walls. After the augmentation procedure, mucoperiostal flaps were repositioned and wound closure was achieved by multiple single sutures (Vicryl 4-0, FS2, Ethicon, Somerville, NJ, USA). Animals received antibiotics for 7 days postoperatively (Streptocillin vet $3-4 \mathrm{ml} /$ animal i.m.; Boehringer Ingelheim, Ingelheim, Germany). Additionally, Temgesic (3-5 ml/animal i.m.; Essex Pharma GmbH, Munich, Germany) was administered for 3 days postoperatively.

\section{Implant placement}

Following a healing period of 12 weeks, surgical sites were exposed by midcrestal incisions and elevation of full-thickness flaps. After identification of the augmented defect sites by the help of previously inserted microscrews, titanium implants (Straumann Bone Level SLActive, Straumann AG, Basel, Switzerland) with dimensions of $3.3 \times 8 \mathrm{~mm}$ were placed in an epicrestal position, according to the manufacturer's surgical protocol. Hereafter, wound closure was obtained by multiple single sutures (Vicryl 4-0, FS2, Ethicon, Somerville, NJ, USA). Postoperatively, digital radiographs were taken to ensure a proper implant installation (Fig. 2d). Animals received antibiotics for 7 days (Streptocillin vet 3-4 ml/ animal i.m.; Boehringer Ingelheim, Ingelheim, Germany). Additionally, Temgesic (3-5 ml/animal i.m.; Essex Pharma GmbH, Munich, Germany) was administered for 3 days postoperatively.

\section{Study termination}

Eight weeks after implant placement, animals were killed by an intracardiac injection of a $20 \%$ solution of pentobarbital (Pentobarbitalnatrium, Apoteket AB; Stockholm, Sweden, $60 \mathrm{mg} / \mathrm{ml}$ ). Following block resection of the hemi-mandibles, augmented/implanted sites were isolated and fixed in $10 \%$ neutral buffered formalin solution for 10 days.

\section{Histological preparation}

The collected specimens were dehydrated using ascending grades of alcohol and xylene, infiltrated, and embedded in methylmethacrylate (MMA, Technovit 9100 NEU, Heraeus Kulzer, Wehrheim, Germany) for non-decalcified sectioning. During this procedure, any negative influence of polymerization heat was avoided due to a controlled polymerization in a cold atmosphere $\left(-4{ }^{\circ} \mathrm{C}\right)$. After $20 \mathrm{~h}$, the specimens were completely polymerized. Each implant site was cut in the buccal-oral direction along with the long axis of the implant using a diamond wire saw (Exakt, Apparatebau, Norderstedt,
Germany), resulting in 4 sections of approximately $300 \mu \mathrm{m}$ in thickness each. Subsequently, all specimens were glued with acrylic cement (Technovit 9100 VLC, Heraeus Kulzer, Wehrheim, Germany) to silanized glass slides (Super Frost, Menzel GmbH, Braunschweig, Germany) and ground to a final thickness of approximately $40 \mu \mathrm{m}$. Prepared specimens were employed for histomorphometric analysis.

\section{Histomorphometrical analysis}

Histological sections were stained with toluidine blue (TB) to evaluate bone formation. The specific staining technique allows the differentiation of mature and new bone according to the color staining strength. Mature bone can be identified by a light blue staining, whereas new bone stains dark blue due to its higher protein content.

Histomorphometrical analysis was performed by one experienced investigator masked to the specific experimental conditions (I.M.). For image acquisition, a color CCD camera (Color ViewTM III, Olympus, Hamburg, Germany) was mounted on a binocular light microscope (Olympus BX50, Olympus, Hamburg, Germany). Digital images (original magnification $\times 200$ ) were evaluated using a software program (analySIS FIVE docu, Soft Imaging System, Münster, Germany).

Histomorphometrical analysis concentrated on two aspects: the defect area (DA) and bone-to-implant contact (BIC). DA was identified at the buccal and oral aspects of the inserted implant being confined by the bottom of the defect, the implant surface, the coronal level of bone in contact with the implant surface (CBI), and the demarcation of the defect and pristine bone at the buccal/oral aspect (Fig. 3a). Within DA, the surface areas of mineralized (MT) and residual $\mathrm{BCP} / \mathrm{NBM}$ particles (BS) were automatically assessed (in \%) by the image analysis software. To assess BIC, the implant shoulder (IS) and CBI were identified. Consequently, BIC was assessed as a percentage of the distance from IS at the buccal aspect to IS at the oral aspect (Fig. 3b). In addition, the vertical linear distance from IS to CBI was assessed at both buccal and oral aspects. Before the start of the histomorphometrical analysis, a calibration procedure was initiated for the image analysis software and revealed that repeated measurements of $n=$ 12 different sections were similar at $>95 \%$ level.

\section{Statistical analysis}

The statistical analysis was performed using a commercially available software program (SPSS Statistics 19.0, SPSS Inc., Chicago, IL, USA). The mean values and standard deviations among animals were calculated for each variable and group. The data rows were examined using the KolmogorovSmirnov test for a normal distribution. Between-group 


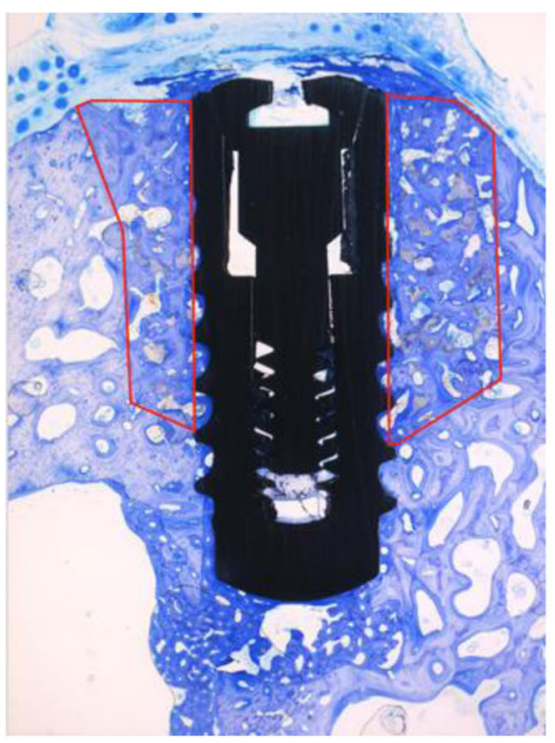

a

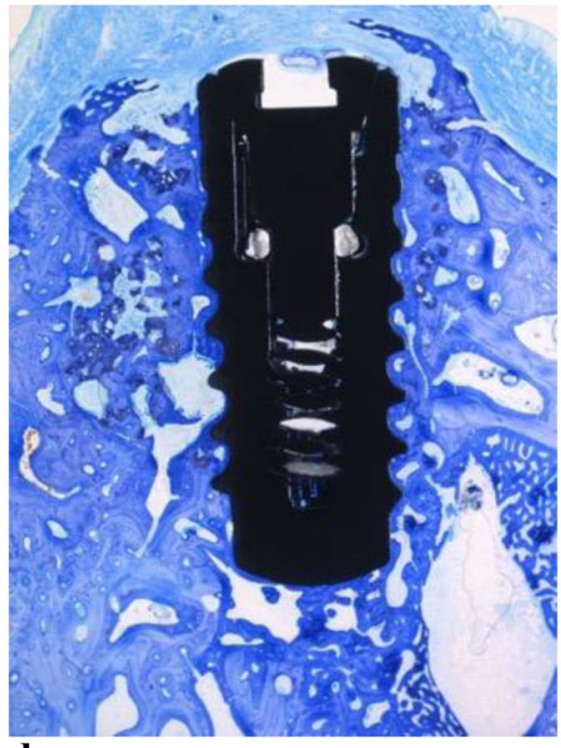

d

Fig. 3 Representative histological views (TB stain, original magnification $\times 4$ ) and histological landmarks. a Defect area (red line) was defined by the implant (line connecting the implant threads), the alveolar crest, and the defect borders (BCP3 specimen). b Red lines

comparisons were performed using the unpaired $t$ test. The alpha error was set at 0.05 .

\section{Results}

\section{Clinical healing}

The postoperative healing was uneventful in all animals. No complications such as allergic reactions, swellings, abscesses, or infections could be observed throughout the whole study period.

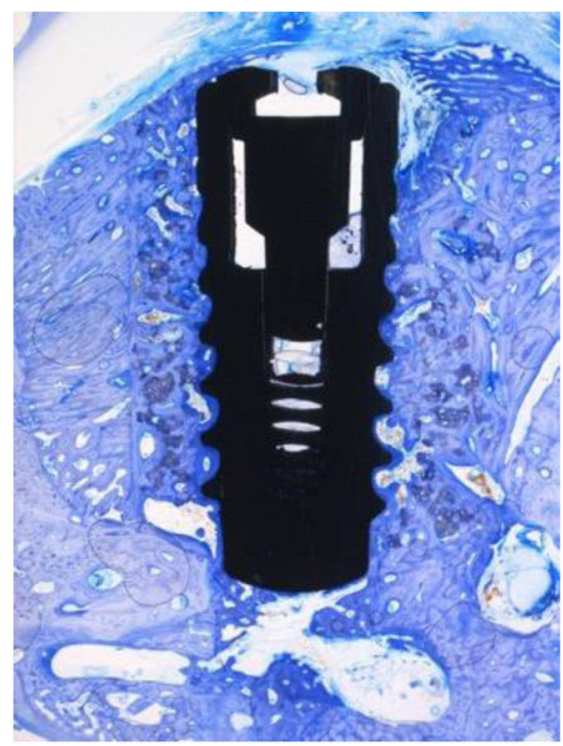

c

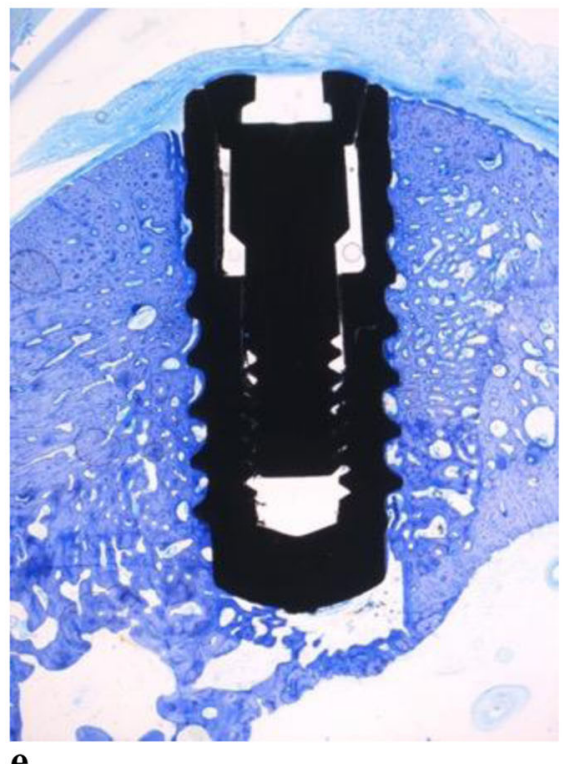

marking IS and CBI which served as reference for the assessment of BIC and IS-CBI values (NBM specimen). c BCP1 specimen. d BCP2 specimen. e Untreated control specimen

\section{Descriptive histology}

After $12+8$ weeks of healing, all histological specimens showed a bony filling of the secluded wound area treated with BCP1-3 and NBM (Fig. 4a). A scaffold of cancellous bone accompanied by blood vessels and bone-forming cells homogeneously surrounded all graft particles. Graft particles of all groups appeared to be in close contact with newly formed bone. However, slight differences between different bone graft materials were visible. NBM particles were well integrated into the surrounding new bone tissue, whereas no signs of graft resorption could be detected (Fig. 4b). In groups 


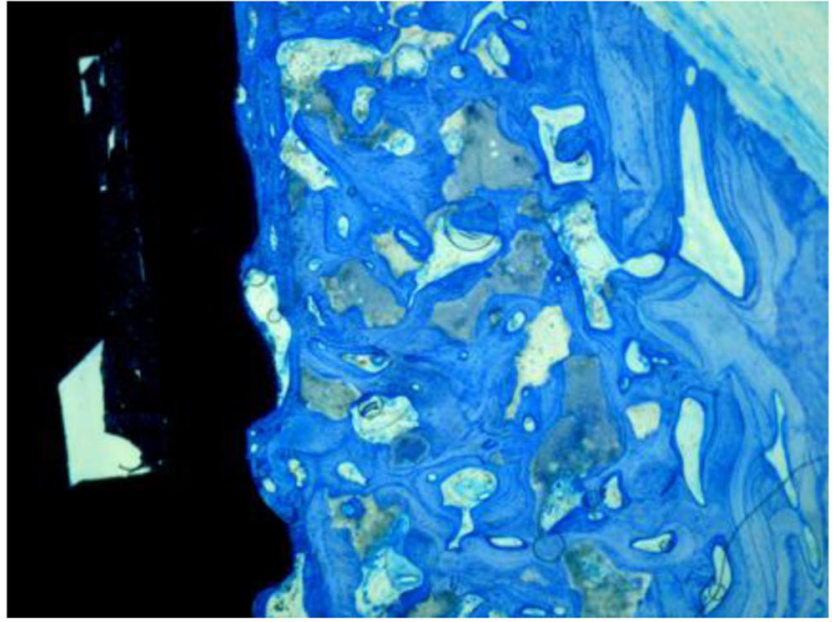

a

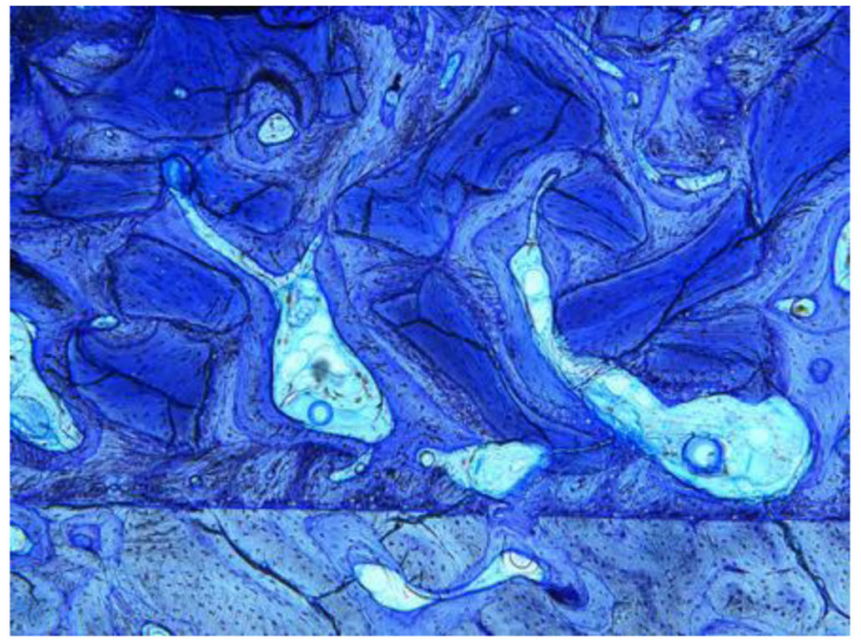

b

Fig. 4 Descriptive histological findings in different groups. a Residual BS could be identified in all groups. These particles were well integrated into the bone matrix $(\mathrm{BCP} 2, \mathrm{~TB}$ stain, original magnification $\times 10)$. $\mathbf{b}$ NBM particles were in close contact with adjacent osteoid tissue but without showing signs of resorption. NBM was well incorporated into the newly formed bone (NBM, toluidine stain, original magnification $\times$

BCP1-3, bone graft particles were more frequently in contact with immature bone revealing increased surface areas lined by a provisional bone matrix. These areas were characterized by a dissolution of BCP, resulting in a superficial disintegration of particles into smaller grains accompanied by osteoclast activity near the graft surface. Disintegrated BCP graft particles were frequently surrounded by newly formed bone (Fig. 4c). Within BCP groups, no pronounced differences regarding resorption pattern and bone remodelling could be noted histologically.

In all groups investigated, titanium implants were lined by a firmly attached mature, parallel-fibered woven bone. Several areas showed implant surfaces being in direct contact with the BCP1-3 or NBM particles (Fig. 4d). However, these areas did not reveal any interposition of NMT between the implant

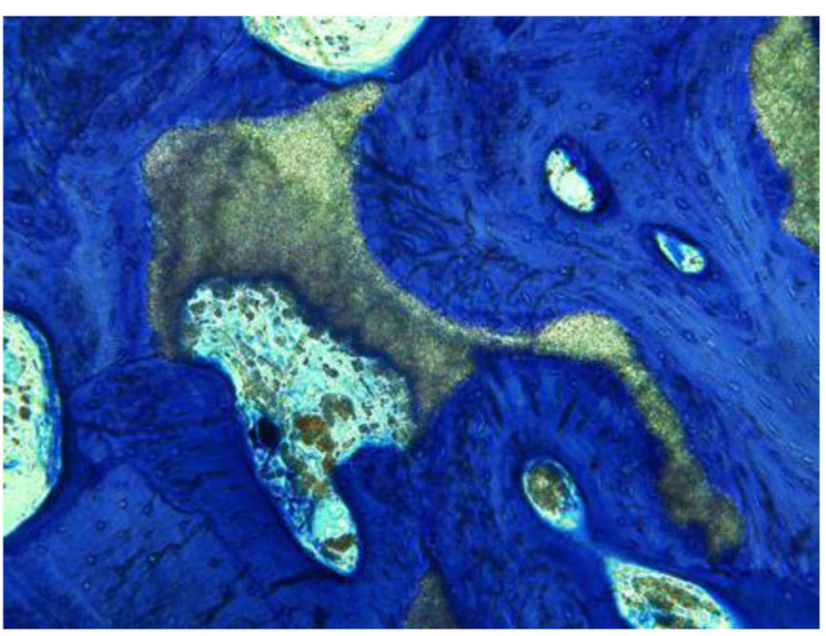

c

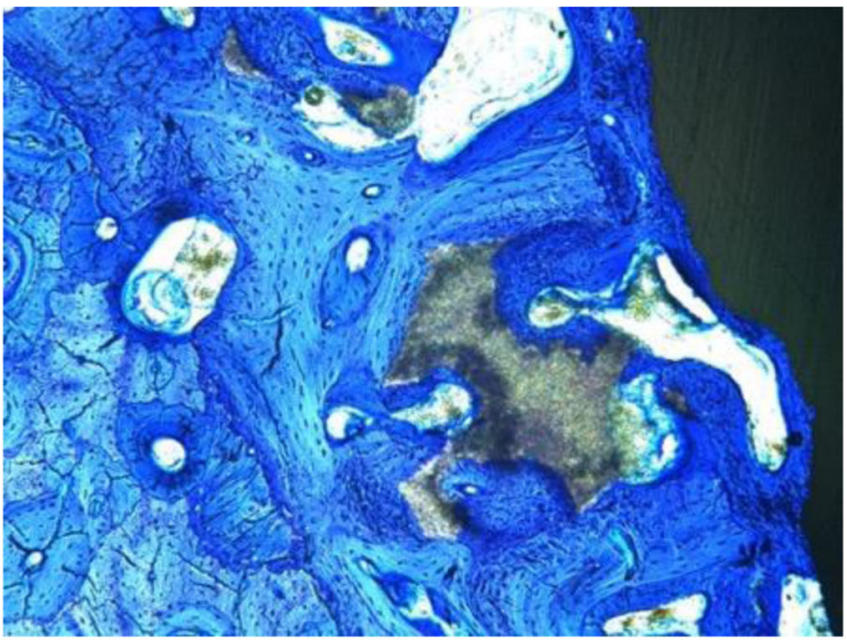

d

20). $\mathbf{c}$ In all BCP groups, bone graft particles were characterized by an ongoing resorption and dissolution accompanied by osteoclast activity near the graft surface $(\mathrm{BCP} 3$, toluidine stain, original magnification $\times$ 50). d All BCP particles were predominantly surrounded by a compact bone matrix, whereas areas with no contact to matured bone were lined by mineralized tissue $(\mathrm{BCP} 3$, toluidine stain, original magnification $\times 40$ )

surface and the respective bone graft particles. The maturity of the woven bone was identifiable by the development of

Table 1 Mean values for MT, NMT, and BSM in all groups $(n=8$ animals)

\begin{tabular}{lcrrrr}
\hline & BCP1 & BCP2 & \multicolumn{1}{l}{ BCP3 } & NBM & \multicolumn{1}{c}{ C } \\
\hline Mean BS (\%) & $9.2 *$ & 14.04 & 13.46 & $21.25^{*}$ & 7.73 \\
SD & 3.28 & 5.77 & 2.76 & 13.52 & 3.23 \\
Mean MT (\%) & 67.49 & 61.75 & 63.06 & 57.36 & 71.63 \\
SD & 14.2 & 9.1 & 10,36 & 10.59 & 14.27 \\
Mean BS + MT (\%) & 76.69 & 75.79 & 76.51 & 78.64 & 79.37 \\
SD & 13.27 & 6.52 & 10.57 & 13.21 & 12.31 \\
\hline
\end{tabular}

* Significant between-group difference at $P<0.05$ 
Table 2 Mean values for BIC and CBI-IS in all groups ( $n=8$ animals)

\begin{tabular}{lrrrrr}
\hline & \multicolumn{1}{c}{ BCP1 } & \multicolumn{1}{c}{ BCP2 } & \multicolumn{1}{c}{ BCP3 } & NBM & \multicolumn{1}{c}{ C } \\
\hline Mean BIC $(\mu \mathrm{m})$ & $18,055.29$ & $15,887.33$ & $17,477.9$ & $17,662.76$ & $16,673.55$ \\
SD & 2176.29 & 4467.02 & 3755.55 & 1430.12 & 2581.41 \\
Mean BIC $(\%)$ & 84.11 & 74.38 & 80.26 & 81.15 & 77.57 \\
SD & 7.84 & 20.5 & 16.13 & 6.23 & 12.87 \\
Mean CBI-ISb $(\mu \mathrm{m})$ & -146.09 & -213.45 & -286.55 & 246.77 & 86.37 \\
SD & 586.16 & 472.94 & 491.34 & 515.75 & 308.18 \\
Mean CBI-ISo $(\mu \mathrm{m})$ & 195.91 & 0 & 16.09 & 64.38 & 139.92 \\
SD & 545.13 & 0 & 50.87 & 72.45 & 258.1 \\
\hline
\end{tabular}

$b$, buccal aspect; $o$, oral aspect primary osteons and appeared to be comparable in regenerated and control areas of all treatment groups.

\section{Histomorphometrical analysis}

Mean values and percentages of MT and BS values in different groups are presented in Table 1. In particular, mean MT
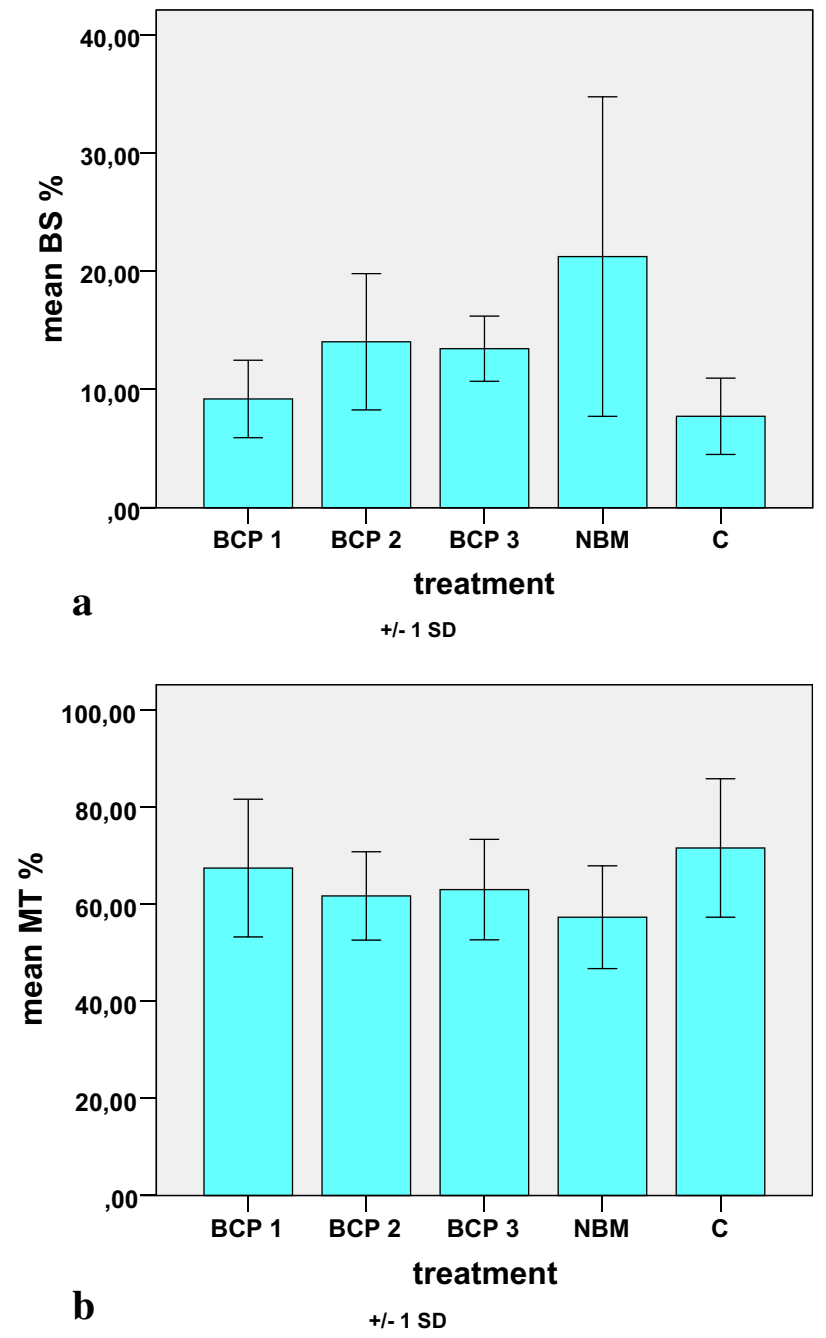

values were equally distributed within the different $\mathrm{BCP}$ groups without showing obvious differences between the respective sites $(61.75 \pm 9.1 \%$ (BCP2) to $67.49 \pm 14.2 \%$ (BCP1)). These percentages were markedly lower in the NBM group $(57.63 \pm 10.59 \%)$, however, without showing any significant differences between the grafted sites $(P>0.05$, respectively).

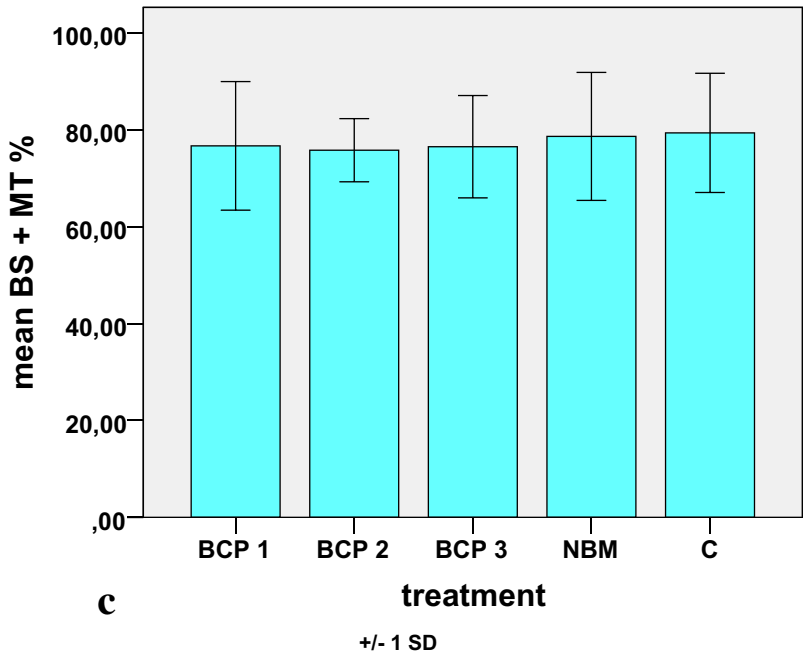

Fig. 5 a Mean bone substitute (BS) in all treatment groups (\%). b Mean mineralized tissue (MT) in all treatment groups (\%). c Mean bone substitute and mineralized tissue (BS+MT) in all treatment groups $(\%)$ 

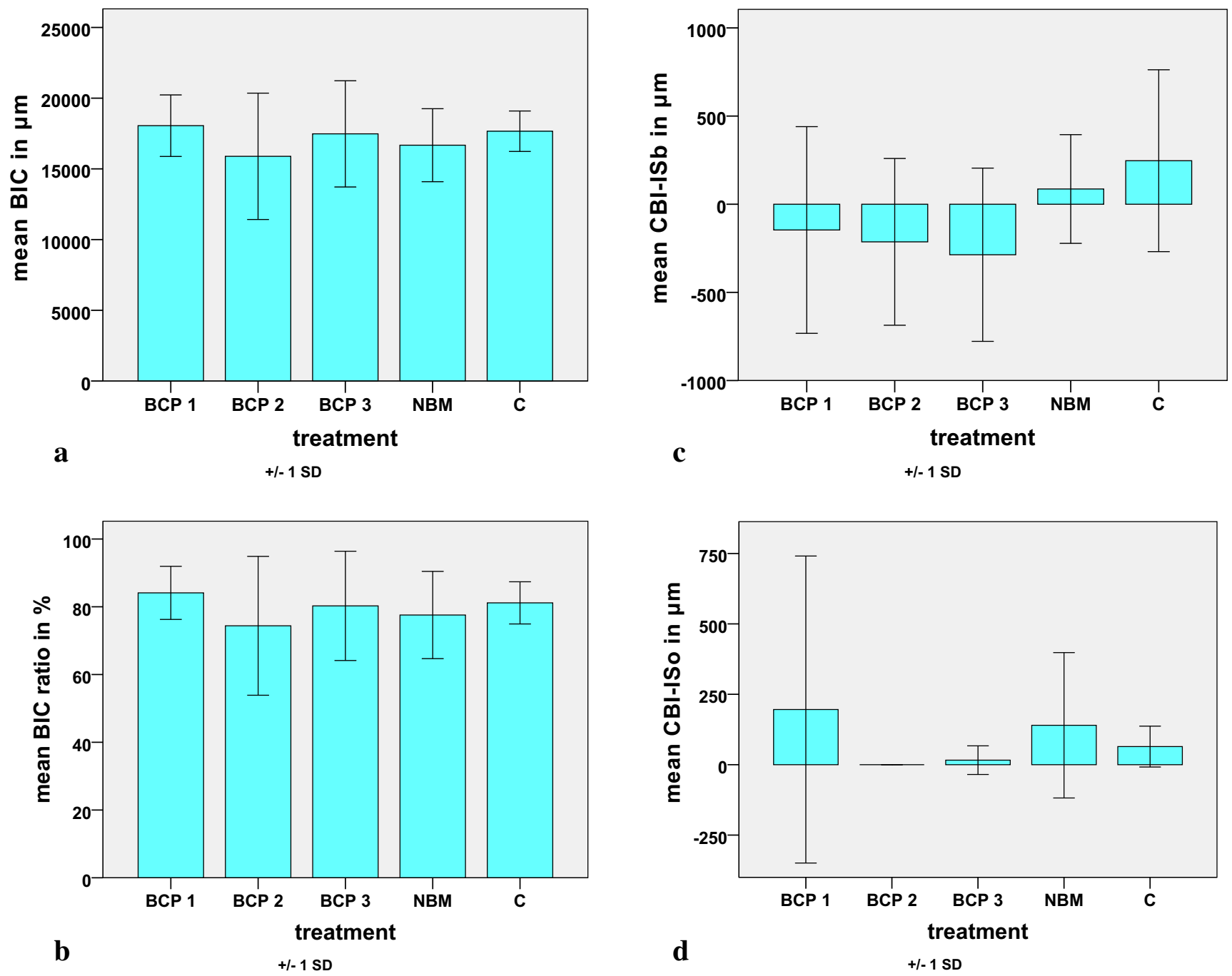

Fig. 6 a Mean bone-to-implant contact (BIC) in all treatment groups $(\mu \mathrm{m})$. b Mean bone-to-implant contact ratio (BIC) in all treatment groups (\%). $\mathbf{c}$ Mean distance between crestal bone level (CBL) and the buccal

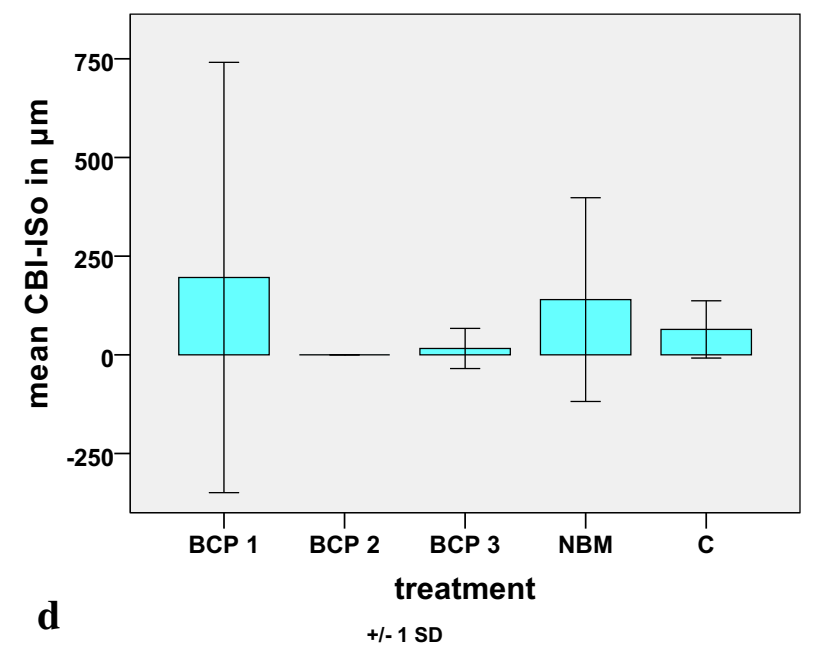

aspect of the implant shoulder (ISb). d Mean distance between crestal bone level (CBL) and the oral aspect of the implant shoulder (ISo)

Mean BS values were highest in the NBM group (21.25 \pm $13.52 \%$ ) and markedly reduced in the different BCP groups (Table 1). The lowest BS values were noted in the BCP1 (9.2 $\pm 3.28 \%$ ) group, reaching statistical significance when compared with the NBM group. When comparing BS + MT values, all grafted areas revealed similar mean values, ranging between $75.79 \pm 6.52 \%(\mathrm{BCP} 2)$ and $78.64 \% \pm 13.21 \%$ (NBM), respectively.

Mean values and percentages of BIC and IS-CBI values in different groups are presented in Table 2. All test and control groups investigated revealed comparable and statistically not significant different $\mathrm{BIC}$ values, ranging from $73.38 \pm 20.5 \%$ (BCP2) to $84.11 \pm 7.84 \%$ (BCP1), respectively. Except for BCP2 grafted sites, all implants investigated were associated with positive IS-CBI values, ranging from $16.09 \pm 50.87 \mu \mathrm{m}$ (BCP3) to $195.91 \pm 545.13 \mu \mathrm{m}$ (BCP1), respectively. These

differences, however, failed to reach statistical significance $(P>0.05$, respectively).

\section{Discussion}

The present study aimed at histologically investigating the influence of three BCP graft materials with different surface topographies on new bone formation and staged osseointegration of titanium implants in minipigs Figs 5 and 6.

The selected experimental model represents a wellestablished standard to investigate bone remodelling processes in implant dentistry [20]. Similar cylindrical-type defects have also been described in previous experimental studies and were reported to be non-critical, as they allowed for a certain spontaneous healing $[4,21]$. 
The present histological analysis has indicated that BCP13 and NBM groups were equally and homogeneously integrated into a newly formed scaffold of cancellous bone after 20 weeks. All bone graft materials lead to comparable amounts of new bone formation, as indicated by comparable mean MT values. Even though mean MT values were markedly lower in the NBM group, these differences did not reach statistical significance when compared with BCP1-3 groups. These differences may be attributed to the higher BS values noted at NBM-treated sites, which in turn reflects a higher degradation rate of $\mathrm{BCP}$ particles. This was particularly noted in the BCP1 group, reaching statistical significance over NBM-treated sites. The dissolution of BCP particles was clearly depicted in the present descriptive histological analysis and was also confirmed in a previous experimental study employing a similar type of microstructured BCP (10\% HA and $90 \% \beta$-TCP, particle size 500-1000 $\mu \mathrm{m}$ ) [21]. The cylindrical-type defects were filled with BCP1, NBM, and another BCP $(60 \%$ HA and 40\% $\beta$-TCP, particle size 500$1000 \mu \mathrm{m})$ (refers to as SBC). At 8 weeks, BCP was associated with significantly higher MT and BS values when compared with NBM- and SBC-treated defects. However, when comparing the histomorphometrical outcomes at 3 and 8 weeks, this BCP also revealed a marked reduction in mean BS values (median 45.5 at 3 weeks and $38.3 \%$ at 8 weeks) [21]. Potential differences between the findings of the latter study and those of the present analysis may be mainly attributed to the prolonged healing period of 20 weeks. Moreover, implant placement and the associated dynamic stages of osseointegration may have also contributed to the dissolution of the various BCP grafts. To the best of our knowledge, these are the first data reporting on the histological outcomes following two-stage implant placement at BCP1-3 grafted sites.

However, previous animal studies performed in the canine tested SBC in dehiscence- and saddle-type defects along with a simultaneous or two-stage grafting procedure $[5,6,22]$. A dissolution of SBC particles was noted between 9 and 10 weeks and resulted in markedly lower BS values than the respective NBM groups, respectively [5, 6, 22]. The pronounced bone remodelling in the SBC group was also evidenced by an elevated collagen type I, osteocalcin, and transglutaminase antigen reactivity in the adjacent nonmineralized tissue [22].

Despite the lack of immunohistochemical analyses, the present analysis also commonly revealed that BCP particles were well integrated and surrounded by bone with an adjacent non-mineralized zone containing multiple dissolved graft particles. Similar findings were made by Dahlin et al. [21] and hypothesized as being linked to a dissolution of ions into the adjacent tissue. In fact, bone remodelling and subsequently resorption of TCP have previously been linked to its microstructure, with a more pronounced activity being noted for submicron-scaled over micron-scaled materials [18, 23].
Accordingly, future studies need to further focus on the specific degradation pattern of BCP1-3.

When further analyzing the present data, it was also noted that BCP1-3 and NBM groups were associated with comparable $\mathrm{BIC}$ values after 8 weeks of healing, which were on a level equivalent to those noted at pristine bone sites. Moreover, the BIC values noted in all test and control groups were within the range of those data $(71.9 \pm 4.0$ to $74.9 \pm 7.8 \%)$ that were reported for the same implant surface after 2 weeks of healing in the mandible of dogs [24, 25].

Previous data also pointed to comparable BIC values following two-stage implant placement at SBC and NBM grafted sites. In particular, after a submerged healing period of 2 weeks, mean BIC values varied between $54.1 \pm 22.6$ and $67.7 \pm 16.9 \%$ in the NBM and between $61.0 \pm 8.7$ and 66.9 $\pm 17.8 \%$ in the SBC group, respectively [6].

\section{Conclusions}

Within the limits of the present study, it was concluded that all investigated bone graft materials supported bone formation and the staged osseointegration of titanium implants.

Acknowledgments We kindly appreciate the skills and commitment of Marcel Obrecht and the team of Lund University. The study materials were kindly provided by Institut Straumann AG.

Funding information Open Access funding provided by Projekt DEAL. This study was funded by Institute Straumann AG, Basel, Switzerland.

\section{Compliance with ethical standards}

Animal selection, management, and surgery protocol were approved by the Malmö-Lund University Animal Experiment Ethics Committee (number M-204-11.7; Malmö-Lund University, Lund, Sweden).

Conflict of interest The authors declare that there are no conflicts of interest.

Ethical approval The article does not contain any studies with human participants.

Informed consent Formal consent was not required.

Clinical relevance All graft materials may be equally suited for clinical application.

Open Access This article is licensed under a Creative Commons Attribution 4.0 International License, which permits use, sharing, adaptation, distribution and reproduction in any medium or format, as long as you give appropriate credit to the original author(s) and the source, provide a link to the Creative Commons licence, and indicate if changes were made. The images or other third party material in this article are included in the article's Creative Commons licence, unless indicated otherwise in a credit line to the material. If material is not included in the article's Creative Commons licence and your intended use is not permitted by statutory regulation or exceeds the permitted use, you will need to obtain 
permission directly from the copyright holder. To view a copy of this licence, visit http://creativecommons.org/licenses/by/4.0/.

\section{References}

1. Cordaro L, Bosshardt DD, Palattella P, Rao W, Serino G, Chiapasco M (2008) Maxillary sinus grafting with Bio-Oss or Straumann Bone Ceramic: histomorphometric results from a randomized controlled multicenter clinical trial. Clin Oral Implants Res 19(8):796803. https://doi.org/10.1111/j.1600-0501.2008.01565.x

2. Haugen HJ, Lyngstadaas SP, Rossi F, Perale G (2019) Bone grafts: which is the ideal biomaterial? J Clin Periodontol 46(Suppl. 21): 92-102. https://doi.org/10.1111/jcpe.13058

3. Lindgren C, Hallman M, Sennerby L, Sammons R (2010) Backscattered electron imaging and elemental analysis of retrieved bone tissue following sinus augmentation with deproteinized bovine bone or biphasic calcium phosphate. Clin Oral Implants Res 21(9):924-930. https://doi.org/10.1111/j.1600-0501.2010.01933.x

4. Jensen SS, Terheyden H (2009) Bone augmentation procedures in localized defects in the alveolar ridge: clinical results with different bone grafts and bone-substitute materials. Int J Oral Maxillofac Implants 24:218-236

5. Schwarz F, Mihatovic I, Golubovic V, Hegewald A, Becker J (2012) Influence of two barrier membranes on staged guided bone regeneration and osseointegration of titanium implants in dogs: part 1. Augmentation using bone graft substitutes and autogenous bone. Clin Oral Implants Res 23(1):83-89. https://doi.org/10.1111/j. 1600-0501.2011.02187.x

6. Mihatovic I, Becker J, Golubovic V, Hegewald A, Schwarz F (2012) Influence of two barrier membranes on staged guided bone regeneration and osseointegration of titanium implants in dogs. Part 2: augmentation using bone graft substitutes. Clin Oral Implants Res 23(3):308-315. https://doi.org/10.1111/j.1600-0501.2011. 02238.x

7. Froum SJ, Wallace SS, Cho SC, Elian N, Tarnow DP (2008) Histomorphometric comparison of a biphasic bone ceramic to anorganic bovine bone for sinus augmentation: 6 - to 8 -month postsurgical assessment of vital bone formation. A pilot study. Int $\mathbf{J}$ Periodontics Restorative Dent 28(3):273-281

8. Rh Owen G, Dard M, Larjava H (2018) Hydoxyapatite/betatricalcium phosphate biphasic ceramics as regenerative material for the repair of complex bone defects. J Biomed Mater Res B Appl Biomater 106:2493-2512. https://doi.org/10.1002/jbm.b. 34049

9. Sanz M, Dahlin C, Apatzidou D, Artzi Z, Bozic D, Calciolari E, De Bruyn H, Dommisch H, Donos N, Eickholz P, Ellingsen JE, Haugen HJ, Herrera D, Lambert F, Layrolle P, Montero E, Mustafa K, Omar O, Schliephake H (2019) Biomaterials and regenerative technologies used in bone regeneration in the craniomaxillofacial region: consensus report of group 2 of the 15th European Workshop on Periodontology on Bone Regeneration. J Clin Periodontol 46(Suppl 21):82-91. https://doi. org/10.1111/jcpe. 13123

10. Nery EB, LeGeros RZ, Lynch KL, Lee K (1992) Tissue response to biphasic calcium phosphate ceramic with different ratios of HA/ beta TCP in periodontal osseous defects. J Periodontol 63(9):729735. https://doi.org/10.1902/jop.1992.63.9.729

11. Habibovic $P$, Yuan $H$, van der Valk CM, Meijer G, van Blitterswijk CA, de Groot K (2005) 3D microenvironment as essential element for osteoinduction by biomaterials. Biomaterials 26:3565-3575. https://doi.org/10.1016/j.biomaterials.2004.09.056
12. Yuan H, van den Doel M, Li S, van Blitterswijk CA, de Groot K, de Bruijn JD (2002) A comparison of the osteoinductive potential of two calcium phosphate ceramics implanted intramuscularly in goats. J Mater Sci Mater Med 13(12):1271-1275. https://doi.org/ 10.1023/A:1021191432366

13. Yuan H, Kurashina K, de Bruijn JD, Li Y, de Groot K, Zhang X (1999) A preliminary study on osteoinduction of two kinds of calcium phosphate ceramics. Biomaterials 20(19):1799-1806. https:// doi.org/10.1016/S0142-9612(99)00075-7

14. Magan A, Ripamonti U (1996) Geometry of porous hydroxyapatite implants influences osteogenesis in baboons (Papio ursinus). J Craniofac Surg 7(1):71-78

15. Habibovic P, Li J, van der Valk CM, Meijer G, Layrolle P, van Blitterswijk CA, de Groot K (2005) Biological performance of uncoated and octacalcium phosphate-coated Ti6Al4V. Biomaterials 26(1):23-36. https://doi.org/10.1016/j.biomaterials. 2004.02.026

16. Le Nihouannen D, Daculsi G, Saffarzadeh A, Gauthier O, Delplace S, Pilet P, Layrolle P (2005) Ectopic bone formation by microporous calcium phosphate ceramic particles in sheep muscles. Bone 36(6):1086-1093. https://doi.org/10.1016/j.bone.2005.02.017

17. Yamasaki H, Sakai H (1992) Osteogenic response to porous hydroxyapatite ceramics under the skin of dogs. Biomaterials 13(5): 308-312. https://doi.org/10.1016/0142-9612(92)90054-R

18. Davison NL, Luo X, Schoenmaker T, Everts V, Yuan H, Barrere-de Groot F, de Bruijn JD (2014) Submicron-scale surface architecture of tricalcium phosphate directs osteogenesis in vitro and in vivo. Eur Cell Mater 27:281-297 discussion 296-7

19. Zhang J, Luo X, Barbieri D, Barradas AM, de Bruijn JD, van Blitterswijk CA, Yuan H (2014) The size of surface microstructures as an osteogenic factor in calcium phosphate ceramics. Acta Biomater 10(7):3254-3263. https://doi.org/10.1016/j.actbio.2014. 03.021

20. Mardas N, Dereka X, Donos N, Dard M (2014) Experimental model for bone regeneration in oral and cranio-maxillo-facial surgery. $\mathrm{J}$ Investig Surg 27(1):32-49. https://doi.org/10.3109/08941939. 2013.817628

21. Dahlin C, Obrecht M, Dard M, Donos N (2015) Bone tissue modelling and remodelling following guided bone regeneration in combination with biphasic calcium phosphate materials presenting different microporosity. Clin Oral Implants Res 26(7):814-822. https://doi.org/10.1111/clr.12361

22. Sager M, Ferrari D, Wieland M, Dard M, Becker J, Schwarz F (2012) Immunohistochemical characterization of wound healing at two different bone graft substitutes. Int J Oral Maxillofac Surg 41(5):657-666. https://doi.org/10.1016/j.ijom.2011.11.017

23. Davison NL, ten Harkel B, Schoenmaker T, Luo X, Yuan H, Everts V, Barrere-de Groot F, de Bruijn JD (2014) Osteoclast resorption of beta-tricalcium phosphate controlled by surface architecture. Biomaterials 35(26):7441-7451. https://doi.org/10.1016/j. biomaterials.2014.05.048

24. Schwarz F, Herten M, Sager M, Wieland M, Dard M, Becker J (2007) Bone regeneration in dehiscence-type defects at chemically modified (SLActive) and conventional SLA titanium implants: a pilot study in dogs. J Clin Periodontol 34(1):78-86. https://doi. org/10.1111/j.1600-051X.2006.01008.x

25. Schwarz F, Ferrari D, Herten M, Mihatovic I, Wieland M, Sager M, Becker J (2007) Effects of surface hydrophilicity and microtopography on early stages of soft and hard tissue integration at non-submerged titanium implants: an immunohistochemical study in dogs. J Periodontol 78(11):2171-2184. https://doi.org/10. 1902/jop.2007.070157

Publisher's note Springer Nature remains neutral with regard to jurisdictional claims in published maps and institutional affiliations. 